\title{
DUAL GENERALIZED BASES IN LINEAR TOPOLOGICAL SPACES
}

\author{
WILLIAM J. DAVIS
}

1. Introduction. Arsove and Edwards in [2] studied generalized bases in linear topological spaces. In this paper, we study a concept which is, in a sense, dual to that of the generalized basis.

Let $X$ be a linear topological space, and let $\left\{x_{\alpha}\right\}$ be a family of elements of that space. Suppose there is a total family of linear functionals, $\left\{\varphi_{\alpha}\right\} \subset X^{*}$, such that $\varphi_{\alpha}\left(x_{\beta}\right)=\delta_{\alpha \beta}$, the Kronecker delta. Then $\left\{x_{\alpha}\right\}$ is called a generalized basis for $X$. If we do not ask that $\left\{\varphi_{\alpha}\right\}$ be a total family, but demand that $\left\{x_{\alpha}\right\}$ be total (i.e. fundamental) in $X$, the resulting biorthogonal system is to be called a dual generalized basis system.

We shall see (example 2) that dual generalized bases do arise rather naturally. Furthermore every locally convex, separable Hausdorff space contains a dual generalized basis. This second result is due to Klee [8]. Arsove and Edwards [2] derived an isomorphism theorem for spaces containing generalized bases which we use to derive a corresponding result for spaces containing dual generalized bases. We also use the Arsove-Edwards theorem to derive isomorphism theorems for quotient spaces of spaces admitting maximal biorthogonal systems.

The author wishes to thank the referee for his helpful suggestions.

2. Classification of maximal biorthogonal systems. Let $X$ be a linear topological space with conjugate space $X^{*}$, and suppose $\left\{x_{\alpha}\right\} \subset X,\left\{\varphi_{\alpha}\right\} \subset X^{*}$ such that $\varphi_{\alpha}\left(x_{\beta}\right)=\delta_{\alpha \beta}$. The system $\left\{x_{\alpha} ; \varphi_{\alpha}\right\}$ is called a biorthogonal system. A second biorthogonal system $\left\{x_{\alpha}^{\prime} ; \varphi_{\alpha}^{\prime}\right\}$ is an extension of the first if $\left\{x_{\alpha} ; \varphi_{\alpha}\right\} \subset\left\{x_{\alpha}^{\prime} ; \varphi_{\alpha}^{\prime}\right\}$. The system $\left\{x_{\alpha} ; \varphi_{\alpha}\right\}$ is maximal if it has no proper extension. We denote the linear span of the family $\left\{x_{\alpha}\right\}$ by $\operatorname{sp}\left\{x_{\alpha}\right\}$, and the closure of this span by $\left[x_{\alpha}\right]$. (This closure is always to be understood in the topology under consideration.) The coefficient map, $\Phi(\cdot)$, takes $X$ in to the space $A$ of all generalized sequences $\left\{a_{\alpha}\right\}$, and is defined by $\Phi(x)=\left\{\varphi_{\alpha}(x)\right\}$.

Dieudonné [3] gives the following criterion for the maximality of a biorthogonal system $\left\{x_{\alpha} ; \varphi_{\alpha}\right\}$ : The system is maximal if and only if (a) $\left[x_{\alpha}\right]^{\perp} \subset\left[\varphi_{\alpha}\right]$, or (b) $\cap_{\alpha} \Re\left(\varphi_{\alpha}\right) \subset\left[x_{\alpha}\right]$. ( $\mathscr{T}\left(\varphi_{\alpha}\right)$ denotes the null space of $\varphi_{\alpha}$ ).

Julia [5] constructed a maximal biorthogonal system in a Hilbert space with the property that both $\mathscr{N}(\Phi)$ and $X \sim\left[x_{\alpha}\right]$ are both infinite

Received by the editors October 4, 1965. 
dimensional. It seems reasonable, however, that the more interesting systems should have at least one of the families, $\left\{x_{\alpha}\right\}$ or $\left\{\varphi_{\alpha}\right\}$, total. A biorthogonal system $\left\{x_{\alpha} ; \varphi_{\alpha}\right\}$ will be called a total system if either $\left[x_{\alpha}\right]=X$ or $\mathscr{l}(\Phi)=\{0\}$. This gives three types of total biorthogonal systems:

(1) $\left\{x_{\alpha} ; \varphi_{\alpha}\right\}$ has both $\left[x_{\alpha}\right]=X$ and $\mathscr{T}(\Phi)=\{0\}$. (Markushevich basis sytem, or complete biorthogonal system, see [2].)

(2) $\left\{x_{\alpha} ; \varphi_{\alpha}\right\}$ has at least $\left[x_{\alpha}\right]=X$.

(3) $\left\{x_{\alpha} ; \varphi_{\alpha}\right\}$ has at least $\mathscr{N}(\Phi)=\{0\}$. (Generalized basis system, see [2].)

Since the second type appears to be dual to the third in terms of the pairing $\left(X, X^{*}\right)$, it is called a dual generalized basis sytem. This duality is more than merely formal, and will be used in deriving the isomorphism theorem in $\$ 4$.

To see that dual generalized bases do occur rather naturally, consider a biorthogonal system $\left\{p_{n}(x) ; w_{n}(x)\right\}$ in $L_{2}(-1,1)$, where $\left\{p_{n}(x)\right\}$ is a simple sequence of polynomials (that is, the degree of $p_{n}(x)$ is precisely $\left.n\right)$. If the sequence $\left\{w_{n}(x)\right\}$ is not total, the system is indeed of type $(2)$, since $\left[p_{n}(x)\right]=L_{2}(-1,1)$ by the Stone-Weierstrass theorem. To construct such an example in $L_{2}(-1,1)$, one may start with the Legendre polynomials, and proceed as in the example of $[2$, p. 100].

3. Minimality and biorthogonality. In a finite dimensional space, a necessary condition that a set of vectors form a basis is linear independence. A natural extension of this condition is also necessary for a family $\left\{x_{\alpha}\right\}$ in an infinite dimensional linear topological space to be a basis. This condition, discussed by Kaczmarz and Steinhaus [6], is called minimality, and is defined as follows: A family $\left\{x_{\alpha}\right\}$ is minimal if no $x_{\alpha}$ is in the closure of the span of the remaining elements of the family. That is, $\left\{x_{\alpha}\right\}$ is minimal if $x_{\alpha} \notin\left[x_{\beta}\right]_{\beta \neq \alpha}$ for each $\alpha$. Minimal sequences in Banach spaces were studied by Frink [4]. A result of his which is generally true for linear topological spaces is:

Lemma. If $\left\{x_{\alpha}\right\}$ is minimal, for any $\alpha$ and any $x \in\left[x_{\alpha}\right]$ there is a unique scalar $a_{\alpha}$ such that $x-a_{\alpha} x_{\alpha} \in\left[x_{\beta}\right]_{\beta \neq \alpha}$.

If $X$ is a Banach space, Frink showed that $\left\{x_{\alpha}\right\}$ is minimal if and only if there exists a family $\left\{\varphi_{\alpha}\right\} \subset X^{*}$ such that the system $\left\{x_{\alpha} ; \varphi_{\alpha}\right\}$ is biorthogonal. This result extends readily to more general linear topological spaces, see, for example $[9$, p. 10]: Let $X$ be a locally convex, Hausdorff linear topological space. A family $\left\{x_{\alpha}\right\}$ in $X$ is mini$\mathrm{mal}$ if and only if there exists a family of continuous linear functionals $\left\{\varphi_{\alpha}\right\}$ such that the system $\left\{x_{\alpha} ; \varphi_{\alpha}\right\}$ is biorthogonal. 
Any biorthogonal system for a locally convex Hausdorff space may be extended to a maximal biorthogonal system. The proof of this assertion amounts to a simple application of Zorn's lemma. It is not true, however, that any minimal family extends to a total family. This amounts to saying just that there is a generalized basis $\left\{x_{\alpha}\right\}$ in some space which is a subset of no total generalized basis for that space. An example of such a generalized basis is the unit vector "basis" for the space $(m)$ of bounded sequences.

If $\left\{x_{\alpha}\right\}$ is a nontotal generalized basis for the locally convex Hausdorff space $X$, the following process may be used to increase the family one element at a time. Let $y \in X \sim\left[x_{\alpha}\right]$. Then, the Hahn-Banach theorem yields a continuous linear functional $y^{*}$ such that $y^{*}(y)=1$ and $y^{*}\left(\left[x_{\alpha}\right]\right)=0$. Let $\left\{\varphi_{\alpha}\right\}$ be the total family of functionals originally associated with $\left\{x_{\alpha}\right\}$. The family $\left\{x_{\alpha}, y\right\}$ is a generalized basis with the family of coefficient functionals $\left\{\varphi_{\alpha}^{\prime}, y^{*}\right\}$ defined by $\varphi_{\alpha}^{\prime}=\varphi_{\alpha}$ $-\left(\varphi_{\alpha}(y)\right) y^{*}$. The biorthogonality of $\left\{x_{\alpha}, y ; \varphi_{\alpha}^{\prime}, y^{*}\right\}$ is clear. We must merely show that the family $\left\{\varphi_{\alpha}^{\prime} ; y^{*}\right\}$ is total. Let $x \in \mathscr{N}\left(\Phi^{\prime}\right)$, where $\Phi^{\prime}$ is the new coefficient mapping. Then $y^{*}(x)=0$ and $\varphi_{\alpha}(x)=0$ for each $\alpha$. Since $\left\{x_{\alpha} ; \varphi_{\alpha}\right\}$ is a generalized basis system, this last equality implies that $x=0$, so that the family $\left\{\varphi_{\alpha}^{\prime}, y^{*}\right\}$ is total. We have, then, this proposition:

Proposition. If $\left\{x_{\alpha}\right\}$ is a nontotal generalized basis for a locally convex, Hausdorff space, $X$, there is a generalized basis $\left\{u_{\alpha}\right\}$ for $X$ such that $\left\{x_{\alpha}\right\} \subset\left\{u_{\alpha}\right\}$, but $\left\{x_{\alpha}\right\} \neq\left\{u_{\alpha}\right\}$.

Every separable, locally convex Hausdorff space contains a dual generalized basis [8]. In fact, every separable Banach space contains a Markushevich basis [10].

4. Isomorphism theorems. A useful concept in the comparison of bases and biorthogonal systems is that of similarity. Arsove [1] calls two bases similar if the same families of expansion coefficients yield convergent expansions in terms of both bases. From this definition he shows that Fréchet spaces having similar bases are themselves isomorphic.

In [2], the definition of similarity is extended as follows: Let $\left\{x_{\alpha} ; \varphi_{\alpha}\right\}$ be a biorthogonal system with $\left\{x_{\alpha}\right\} \subset X$, and $\left\{y_{\alpha} ; \psi_{\alpha}\right\}$ a biorthogonal system with $\left\{y_{\alpha}\right\} \subset Y$. Let $\Phi$ and $\Psi$ be the corresponding coefficient mappings. Then, $\left\{x_{\alpha} ; \varphi_{\alpha}\right\}$ and $\left\{y_{\alpha} ; \psi_{\alpha}\right\}$ are similar if $\Phi(X)=\Psi(Y)$. From this they deduce the important isomorphism theorem which we shall need in the following. 
Theorem (ARsove-Edwards). Let $X$ and $Y$ be complete metric linear spaces containing similar generalized bases $\left\{x_{\alpha}\right\}$ and $\left\{y_{\alpha}\right\}$ respectively. Then, there is an isomorphism $T$ of $X$ onto $Y$ such that $T\left(x_{\alpha}\right)=y_{\alpha}$.

The known converse of this theorem is true in the more general form: Let $X$ and $Y$ be linear topological spaces isomorphic under the map $T$. If $\left\{x_{\alpha}\right\} \subset X$ is a Schauder basis (Markushevich basis, generalized basis, dual generalized basis), then the family $\left\{y_{\alpha}\right\}$ defined by $T x_{\alpha}=y_{\alpha}$ is also a Schauder basis (etc.) for $Y$.

Using the isomorphism theorem, we get an isomorphism for certain quotient spaces of complete metric linear spaces, which contain similar maximal biorthogonal systems. First we need the lemma:

Lемма 2. Let $\left\{x_{\alpha} ; \phi_{\alpha}\right\}$ be a biorthogonal system for the linear topological space $X$. Let $Q$ denote the quotient map from $X$ to $X / \Re(\Phi)$.

(i) $\left\{Q\left(x_{\alpha}\right) ; \tilde{\phi}_{\alpha}\right\}$ is a generalized basis for $X / \mathfrak{N}(\Phi)$, where $\tilde{\phi}_{\alpha}$ is defined by $\tilde{\phi}_{\alpha}(Q(x))=\phi_{\alpha}(x)$.

(ii) If $\left\{x_{\alpha} ; \phi_{\alpha}\right\}$ is a dual generalized basis, then $\left\{Q\left(x_{\alpha}\right) ; \tilde{\phi}_{\alpha}\right\}$ is a Markushevich basis.

(iii) If $\left\{x_{\alpha} ; \phi_{\alpha}\right\}$ is a maximal biorthogonal system for a locally convex, Hausdorff space $X$, then $\left\{Q\left(x_{\alpha}\right) ; \tilde{\phi}_{\alpha}\right\}$ is a Markushevich basis for $X / \mathscr{N}(\Phi)$ if and only if $\left\{x_{\alpha} ; \phi_{\alpha}\right\}$ is a dual generalized basis system for $X$.

Proof. Let $A$ denote the range of $\Phi$. Define the map $\tilde{\Phi}: X / \mathfrak{N}(\Phi)$ $\rightarrow A$ by $\tilde{\Phi} \circ Q=\Phi$. Then, topologize $A$ with the topology induced from $X / \mathscr{N}(\Phi)$ by $\tilde{\Phi}$. With this topology, $\Phi$ must be continuous. Further $\tilde{\Phi}$ is the coefficient map associated with the continuous linear functionals, $\left\{\tilde{\phi}_{\alpha}\right\}$. It is clear that $\mathscr{N}(\tilde{\Phi})=\{0\}$, proving (i). If $\left\{x_{\alpha}\right\}$ is total, then so is $\left\{Q\left(x_{\alpha}\right)\right\}$, so we have (ii). For (iii), assume that $\left\{Q\left(x_{\alpha}\right)\right\}$ is total. From the maximality of the system $\left\{x_{\alpha} ; \phi_{\alpha}\right\}$, we have $\mathscr{T}(\Phi)$ $C\left[x_{\alpha}\right]$, so if $f \in\left[x_{\alpha}\right]^{\perp}$, then $f \in(\mathscr{N}(\Phi))^{\perp}$. Therefore, $\tilde{f}$ induces a map $f: X / \Re(\Phi) \rightarrow A$ defined by $f=\tilde{f} \circ Q$. Therefore, $\tilde{f}\left(Q\left(x_{\alpha}\right)\right)=f\left(x_{\alpha}\right)=0$, and the totality of $\left\{Q\left(x_{\alpha}\right)\right\}$ gives us $\tilde{f}=0$. Therefore, $f=0$ and $\left\{x_{\alpha}\right\}$ is indeed a total family.

Theorem 1. Let $X$ and $Y$ be complete metric linear spaces. Let $\left\{x_{\alpha} ; \varphi_{\alpha}\right\}$ and $\left\{y_{\alpha} ; \psi_{\alpha}\right\}$ be similar biorthogonal systems for $X$ and $Y$ respectively. Then, there is an isomorphism $T: X / \mathfrak{T}(\Phi) \rightarrow Y / \mathfrak{N}(\Psi)$ such that $T\left(Q x_{\alpha}\right)=Q^{\prime} y_{\alpha},\left(Q\right.$ and $Q^{\prime}$ are the quotient maps involved.)

The proof follows from the lemma and the Arsove-Edwards isomorphism theorem.

Lemma 3. Let $\left\{x_{\alpha} ; \phi_{\alpha}\right\}$ be a biorthogonal system for a space $X$. Let 
$\chi: X \rightarrow X^{* *}$ be the canonical map. If the biorthogonal system is a dual generalized basis sytem for $X$, then $\left\{\phi_{\alpha} ; \chi\left(x_{\alpha}\right)\right\}$ is a generalized basis for $X^{*}$.

Note. The dual form of this lemma does not hold: Let $\left\{e_{n}\right\}$ be the "unit vector basis" for the spaces $(l)$ and $(m)$. Let $\chi_{1}:(l) \rightarrow\left(m^{*}\right)$ and $\chi_{m}:(m) \rightarrow\left(m^{* *}\right)$ be the canonical embeddings. Then $\left\{e_{n} ; \chi_{1}(e n)\right\}$ is a generalized basis for $(m)$, but $\left\{\chi_{1}\left(e_{n}\right) ; \chi_{m}\left(e_{n}\right)\right\}$ is not even a maximal system.

Proof. Let $\left\{x_{\alpha} ; \varphi_{\alpha}\right\}$ be a dual generalized basis sytem. Let $f \in \bigcap_{\alpha} \Re\left(x_{\alpha}\right)$. Then, $f\left(x_{\alpha}\right)=0$ for all $\alpha$, so $f(u)=0$ for each $u \in X$. That is, $f=0$, so $\left\{\varphi_{\alpha} ; \hat{x}_{\alpha}\right\}$ is a generalized basis sytem for $X^{*}$.

We now define the dual form of similarity: Let $X$ and $Y$ be linear topological spaces. Let $\left\{x_{\alpha} ; \varphi_{\alpha}\right\}$ and $\left\{y_{\alpha}: \psi_{\alpha}\right\}$ be biorthogonal systems for $X$ and $Y$, respectively. If $\hat{X}$ and $\hat{Y}$ are the coefficient maps associated with the families $\left\{\hat{x}_{\alpha}\right\}$ and $\left\{\hat{y}_{\alpha}\right\}$, the systems $\left\{x_{\alpha} ; \varphi_{\alpha}\right\}$ and $\left\{y_{\alpha} ; \psi_{\alpha}\right\}$ are said to be ${ }^{*}$-similar if $\hat{X}\left(X^{*}\right)=\hat{Y}\left(Y^{*}\right)$. Using this definition, the following lemma is a corollary of the Arsove-Edwards theorem.

Lemma 4. Let $X$ and $Y$ be linear topological spaces with metric topologies on $X^{*}$ and $Y^{*}$ making them complete. If $\left\{x_{\alpha} ; \varphi_{\alpha}\right\}$ and $\left\{y_{\alpha} ; \psi_{\alpha}\right\}$ are ${ }^{*}$-similar dual generalized bases, then there is an isomorphism $\mathrm{J}$ of $X^{*}$ onto $Y^{*}$ such that $\Im\left(\varphi_{\alpha}\right)=\psi_{\alpha}$.

With this lemma, we have the machinery to prove the isomorphism theorem.

Theorem 2. Let $X$ and $Y$ be Banach spaces, and let $\left\{x_{\alpha} ; \phi_{\alpha}\right\}$, $\left\{y_{\alpha} ; \psi_{\alpha}\right\}$ be ${ }^{*}$-similar dual generalized basis for $X$ and $Y$ respectively. Then, $X$ and $Y$ are isomorphic under a map $T$ satisfying $T\left(x_{\alpha}\right)=y_{\alpha}$.

Proof. By Lemma 4, there is an isomorphism $S: Y^{*} \rightarrow X^{*}$ with $S\left(\psi_{\alpha}\right)=\phi_{\alpha}$. We let $\left\{\hat{x}_{\alpha}\right\}$ and $\left\{\hat{y}_{\alpha}\right\}$ denote the canonical images of $\left\{x_{\alpha}\right\}$ and $\left\{y_{\alpha}\right\}$ in the second conjugates $X^{* *}$ and $Y^{* *}$. For any $\psi \in Y^{*}$, we have $\left(\left(S^{*}\left(\hat{x}_{\alpha}\right)-\hat{y}_{\alpha}\right) \psi=\hat{x}_{\alpha}(S(\psi))-\hat{y}_{\alpha}(\psi)=0\right.$ from the definition of the isomorphism $S$ (see [2; p. 100]). Thus, $S^{*}\left(\hat{x}_{\alpha}\right)=\hat{y}_{\alpha}$, and we must have $U\left[\hat{x}_{\alpha}\right]=\left[\hat{y}_{\alpha}\right]$ under the restriction, $U$, of $S^{*}$ to $\left[\hat{x}_{\alpha}\right]$. Therefore, if $\chi_{X}$ and $\chi_{Y}$ are the canonical embeddings, the desired isomorphism is given by $T=\chi_{\bar{Y}}^{-1} \circ U \circ \chi_{X}$.

The property of Banach spaces that makes this theorem valid is the fact that the canonical maps are isomorphisms.

5. Related results. If $\left\{x_{\alpha} ; \varphi_{\alpha}\right\}$ is a generalized basis system for a locally convex space $X$, the family $\left\{\varphi_{\alpha}\right\}$ is uniquely determined if 
and only if $\left\{x_{\alpha}\right\}$ is total. A similar situation holds for dual generalized basis systems.

THEOREM 3. Let $\left\{x_{\alpha} ; \varphi_{\alpha}\right\}$ be a dual generalized basis system for the linear topological space $X$. The family $\left\{x_{\alpha}\right\}$ is uniquely determined if and only if $\left\{\varphi_{\alpha}\right\}$ is total.

Proof. Suppose $\left\{\varphi_{\alpha}\right\}$ is not total, and let $u \in \mathscr{N}(\Phi)$ such that $u \neq 0$. Let $x_{\alpha}^{\prime}=x_{\alpha}+e_{\alpha} u$, where $e_{\alpha}$ vanishes except for a finite number of indices, $\alpha_{j}$, and then $e_{\alpha_{j}}=1$. Let $A^{\prime}$ be the index set for $\left\{x_{\alpha}\right\}$ with the $\alpha_{j}^{\prime}$ 's removed. The family $\left\{x_{\alpha}^{\prime}\right\}$ is clearly total if the element $u$ is in $\left[x_{\alpha}\right]_{\alpha \in A^{\prime}}$. By the lemma of Frink's mentioned in $\S 3$, the element $u-\sum_{j=1}^{n} \varphi_{\alpha_{j}}(u) x_{\alpha_{j}}$ is in $\left[x_{\alpha}\right]_{\alpha \in A^{\prime}}$. The sum involved vanishes since $u \in \mathfrak{N}(\Phi)$. Thus, $u \in\left[x_{\alpha}\right]_{\alpha \in A^{\prime}}$, so $\left\{x_{\alpha}^{\prime}\right\}$ is total. This proves that $\left\{x_{\alpha}^{\prime} ; \varphi_{\alpha}\right\}$ is a dual generalized basis. The converse is immediate.

If the spaces $X$ and $Y$ are "nice" enough (for example, Banach spaces) a generalized basis for $X$ which is not total cannot be simultaneously similar and *-similar to a noncomplete dual generalized basis in $Y$.

Proposition 3. Let $X$ and $Y$ be Banach spaces. Let $\left\{x_{\alpha} ; \varphi_{\alpha}\right\}$ be a generalized basis system for $X$ which is simultaneously similar and *similar to the dual generalized basis system $\left\{y_{\alpha} ; \psi_{\alpha}\right\}$ for $Y$. Then $X$ and $Y$ are isomorphic under $T$ with $T x_{\alpha}=y_{\alpha}$ and both systems are Markushevich basis systems.

Proof. According to similarity, Lemma 2 and Theorem 1, there is an isomorphism $\widetilde{T}$ from $X=X / \mathfrak{N}(\Phi)$ to $Y / \mathscr{N}(\Psi)$. By Lemma 2, if $Q^{\prime}: Y \rightarrow Y / \mathscr{T}(\Psi)$, the family $\left\{Q^{\prime}\left(y_{\alpha}\right)\right\}$ is a Markushevich basis for $Y / \mathscr{N}(\Psi)$. Since $\widetilde{T}$ is an isomorphism, and since $\widetilde{T}^{-1}\left(Q^{\prime}\left(y_{\alpha}\right)\right)=x_{\alpha}$, the family $\left\{x_{\alpha}\right\}$ is a Markushevich basis for $X$. Using the ${ }^{*}$-similarity and Theorem 2, there is an isomorphism $T: X \rightarrow Y$, such that $\left\{T x_{\alpha}=y_{\alpha}\right\}$ is a Markushevich basis for $Y$.

\section{REFERENCES}

1. M. G. Arsove, Similar bases and isomorphisms in Frechet spaces, Math. Ann. 135 (1958), 283-293.

2. M. G. Arsove and R. E. Edwards, Generalized bases in topological linear spaces, Studia Math. 19 (1960), 95-113.

3. J. Dieudonné, On biorthogonal systems, Michigan Math J. 2 (1954), 7-20.

4. O. Frink, Jr., Series expansions in linear vector space, Amer. J. Math. 63 (1941), 87-100.

5. G. Julia, Exemples de structure des systèmes duaux de l'espace hilbertien,

C. R. Acad. Sci. Paris 216 (1943), 465-468.

6. S. Kaczmarz and H. Steinhaus, Orthogonalreihen, Warsaw, 1935. 
7. J. L. Kelley, I. Namioka et al., Linear topological spaces, Princeton, N. J., 1963.

8. V. L. Klee, On the Borelian and projective types of linear subspaces, Math. Scand. 6 (1958), 189-199.

9. N. Bourbaki, Les structures fondamentales de l'analyse, Livre V: Espaces vectoriels topologique, Actualités Sci. Ind. No. 1189, Hermann, Paris, 1953.

10. A. Markushevich, Sur les bases (au sens large) dans les espaces linéaires, Dokl. Akad. Nauk 41 (1943), 227-229.

The OHio State University 